\title{
Efficacy and Safety of Sutureless Trabeculectomy with Using Porous Collagen (Ologen) in Management of Glaucoma Patients
}

\author{
Ahmed Elbably ${ }^{1}$, Haytham Rezq ${ }^{1}$, Mohamed ElDakkak ${ }^{1}$, Anastasios E Sepetis ${ }^{1}$, Tageldin M Othman ${ }^{2}$ \\ ${ }^{1}$ Department of Ophthalmology, Southampton University Hospital NHS Foundation Trust, Southampton, UK \\ ${ }^{2}$ Department of Ophthalmology, Faculty of Medicine, Aswan University, Aswan, Egypt
}

Purpose: This study aims to investigate the safety, efficacy and feasibility of sutureless trabeculectomy using a porous collagen matrix that contains a connected porous structure (Ologen) in the control of intraocular pressure (IOP) in glaucoma patients.

Methods: The study includes 25 eyes from 24 patients that met the inclusion criteria. All eyes underwent trabeculectomy with the Ologen implant that provides a space with a dynamic and physiological aqueous reservoir system. The operation was considered successful if IOP is $<15 \mathrm{mmHg}$ without need of IOP-lowering drops at 18 months of follow-up.

Results: The target IOP was achieved in 21 out of the 25 eyes (84\%), the remaining four eyes all had an IOP of $16 \mathrm{mmHg}$ at 18 months. Short-term complications consisted of seven cases of bleb leakage treated with bandage contact lens and one case of mild choroidal effusion which resolved after observation alone.

Conclusions: We can conclude that this technique can provide safety, effectiveness and short learning curve for ophthalmology trainees with lower incidence of perioperative and postoperative complications. Further studies may be required to prove stability and long-term efficacy in management of glaucoma patients.

Key Words: Glaucoma, Ologen, Trabeculectomy

Although trabeculectomy was first introduced more than four decades ago, it remains the gold standard primary surgical procedure to lower the intraocular pressure (IOP) in patients with glaucoma. In various studies, the success rate has ranged from $76 \%$ to $87 \%[1-8]$. However, the risk of complications and failure are still an issue to be ad-

Received: March 19, 2021 Final revision: June 17, 2021

Accepted: June 18, 2021

Corresponding Author: Tageldin M Othman, MD, FRCS (Glasgow). Department of Ophthalmology, Faculty of Medicine, Aswan University, Sahari, Airport Road, Aswan 81528, Egypt. Tel: 20-106-123-4849, Fax: 20-97-3480-449, E-mail: tageldin1973@gmail.com dressed. Several studies have highlighted a variety of risk factors that can compromise the success rate of this procedure. This includes age below 40 years at the time of surgery, black race, presence of systemic disease such as diabetes mellitus, IOP greater than $40 \mathrm{mmHg}$ along the course of the disease, the prolonged use of topical IOP-lowering medications before surgery, previous history of argon laser trabeculoplasty, pseudophakia or aphakia, and previous ocular surgery $[3,4,7,8]$.

Moreover, it is recognised that some types of glaucomas are associated with increased risk of failure of trabeculectomies such as the secondary glaucomas (steroid-induced, neovascular, uveitic, angle recession), pseudophakia, and 
aphakia $[5,6,9]$. On the other hand, several surgical techniques have been associated with reduced failure rate, including superonasal location of scleral flap [10] and intraoperative use of mitomycin C [2-4,8,11]. However, the type of conjunctival flap isn't known to affect the success rate, as some studies showed better success rates with limbus-based conjunctival flap compared to fornix-based, while others reported similar success rates between the two [12].

Postoperatively, the use of subconjunctival 5-fluorouracil injection and the performance of laser suture lysis has been associated with improved success rates $[4,12]$. Therefore, identifying the risk factors for trabeculectomy failure will aid clinicians to institute prompt intervention within the perioperative period to increase chances for success. It will also help clinicians during the preoperative counselling of patients regarding outcomes of surgery.

Recently, biomedical devices to reduce the risk of scar formation were introduced by tissue engineering and have been proved to modify the process of wound healing. Ologen (Ologen; Aeon Astron Europe, Leiden, The Netherlands), a new product that modulates wound healing, is a 3D collagen-glycosaminoglycan scaffold specifically designed to promote wound healing with minimal scarring in a wide range of ophthalmic surgeries. It has been used to create a healthy vascular bleb following trabeculectomy.

Ologen is a porous collagen matrix in a dry form of scaffold that contains a connected porous structure of 10 to $300 \mu \mathrm{m}$ diameter made of cross-linked lyophilized porcine type I atelocollagen ( $\geq 90 \%)$ and glycosaminoglycans $(\leq 10 \%)$. The collagen matrix is thought to eventually biodegrade within 90 to 180 days. Random orientation of collagen generates the 3-D porous structure which interrupts the alignment of fibroblasts and therefore enables a random pattern of fibroblast deposition, not suppressing or inhibiting fibroblast growth but guiding fibroblasts to grow randomly inside the matrix. Modulating fibroblast behavior is thought to produce a more physiological environment without scar formation.

\section{Materials and Methods}

\section{Case selection and regulatory approval}

Twenty-five eyes, each diagnosed with advanced glaucoma (either primary or secondary) with medically uncontrolled IOP were recruited to the current study, and were assigned to the study intervention. The study patients were recruited after the approval of the ethics committee of Magrabi Eye Hospital in Riyadh, Kingdom of Saudi Arabia (MEHR 114-17). Informed consent was obtained from all patients. Recruited patients all had uncontrolled IOP in the study eye with maximal tolerable medical therapy. Uncontrolled glaucoma was defined as uncontrolled IOP $(\geq 22 \mathrm{mmHg}$ ) measured with a Goldmann applanation tonometer with visual field defect progression on maximal medical IOP-lowering treatment. Exclusion criteria were known allergy to collagen and patient refusal. For each patient, preoperative, intraoperative, and postoperative data were collected.

\section{1) Preoperative characteristics}

Preoperative characteristics were age, sex, type of glaucoma, IOP, and number of preoperative glaucoma medications used during a period of three months before surgery. Patients also underwent angle examination using gonioscopy, automated perimetry (visual field testing) and fundus examination. Following surgery, all these examinations, apart from the visual field, were performed on the first day and then repeated after one week as well as after 1, 3, 6, 9, 12 , and 18 months. Visual field tests were repeated every 6 months.

\section{2) Intraoperative characteristics}

Intraoperative characteristics were date of operation, surgical technique, and presence of any intraoperative complications.

\section{3) Postoperative characteristics}

IOP, complications, and number of postoperative glaucoma medications were recorded. All IOP measurements were performed using the Goldmann applanation tonometer.

\section{Primary study outcome}

The primary study outcome was defined as a post-trabeculectomy IOP $<15 \mathrm{mmHg}$ without glaucoma medication 

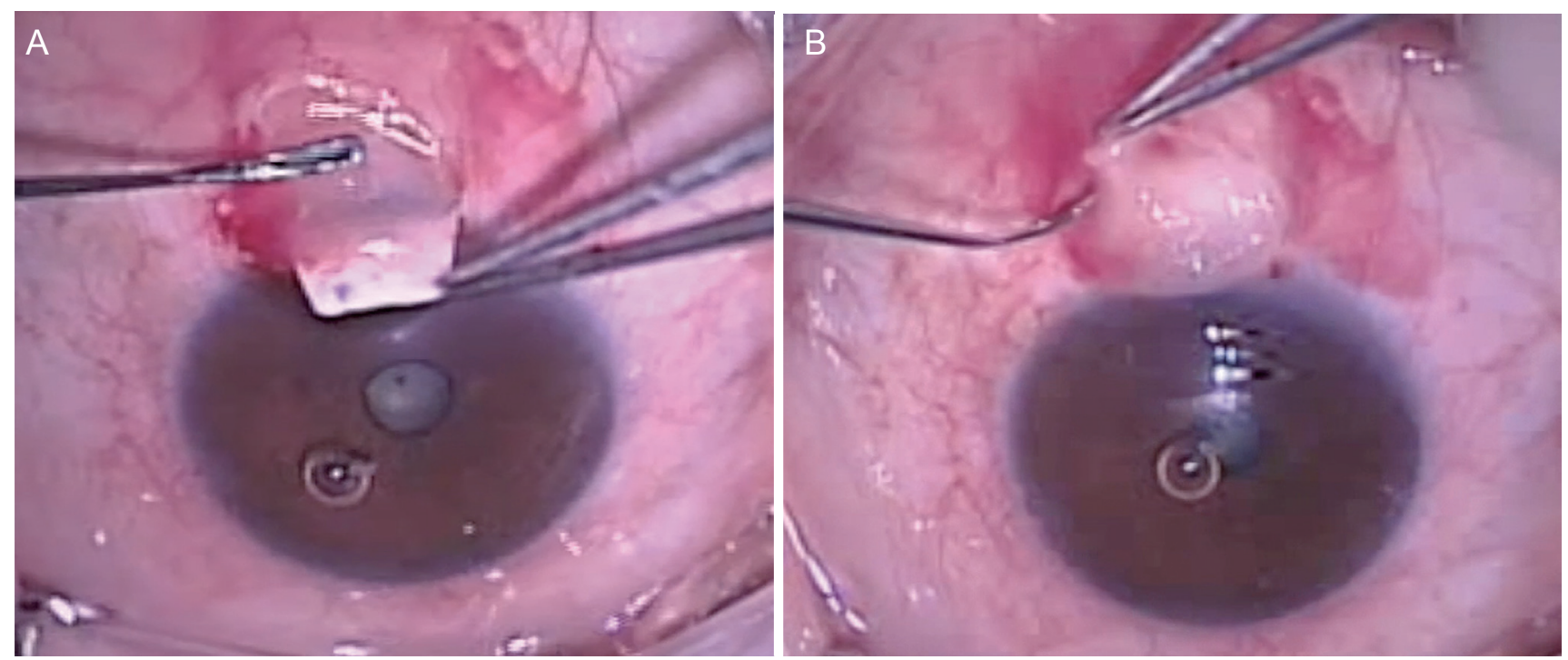

Fig. 1. (A) First layer of Ologen under scleral flab. (B) Second layer of Ologen above scleral flab. Informed consent for publication of the clinical images was obtained from the patient. Informed consent for publication of the clinical images was obtained from the patient.

and a qualified success when IOP was $<15 \mathrm{mmHg}$ with glaucoma medication. It was considered a failure when IOP was $\geq 15 \mathrm{mmHg}$ with glaucoma medication, or when an eye required further glaucoma drainage surgery.

\section{Secondary study outcome}

Secondary study outcomes included the number of postoperative glaucoma medications, postoperative complications, and number of needling procedures required in each group. Complications were considered as follows: hypotony was considered when a postoperative IOP $<5 \mathrm{mmHg}$ for more than 2 weeks. The anterior chamber depth was clinically compared with the fellow eye. Anterior chamber was shallow when a peripheral iridocorneal touch occurred and flat with a lenticulo corneal touch. Anterior chamber flare would indicate anterior chamber inflammation. Choroidal detachment would be present when seen in the peripheral retina by an indirect ophthalmoscope.

\section{Surgical procedures: sutureless scleral flap trabeculec- tomy with mitomycin-C and Ologen}

A fornix-based conjunctival flap is performed, then; creating a $3 \times 4-\mathrm{mm}$ rectangular one third thickness scleral flap, mitomycin-C in a concentration of $0.2 \mathrm{mg} / \mathrm{mL}$ was applied for 2 minutes, $2 \times 2 \mathrm{~mm}$. An internal sclerostomy is commonly created with the use of a Kelly glaucoma punch (Geuder, EA/1, 19 gauge, $1.0 \mathrm{~mm}$ ).

An iridectomy is performed to reduce the risk of iris occluding the sclerostomy, double-layered Ologen sandwiching the scleral flap, we cut one Ologen disc into two halves, and then we put one half under the scleral flab (Fig. 1A) and the other half above it (Fig. 1B). As Ologen is a porous collagen, it has relatively rough surface (not slippery) and it swells once soaked by BSS that is why it stays perfectly in its position. Suture less closure of the scleral flap, conjunctiva is secured at the limbus. The conjunctival closing was performed via two main cardinal (limbal) 10/0 nylon sutures and one 10/0 vicryl mattress in-between.

\section{Statistical analyses}

All statistical analysis was performed using GraphPad Prism ver. 8.4.1 (GraphPad Software, San Diego, CA, USA). Gaussian distribution was tested for all values using the D' Agostino and Pearson test. Where normality tests were not passed, median values and interquartile range are reported. Two-tailed Wilcoxon matched-pairs signed rank tests were used to for comparison between postoperative and the preoperative values. For all analyses, a $p$-value of less than 0.05 was considered to be statistically significant. 


\section{Results}

We identified 25 eyes from 24 patients that met the inclusion criteria. There were 17 males and seven females with median age (interquartile range, IQR) at the date of the operation 54 years (range, 15-73 years). Five eyes had previous trabeculectomy and 18 were phakic. Baseline characteristics are described in Table 1. The median (IQR)

Table 1. Comparison of baseline characteristic among two groups

\begin{tabular}{ll}
\hline Characteristics & Value \\
\hline No. of patients & 24 \\
No. of eyes & 25 \\
Sex, male & $17(79.8)$ \\
Age (yr), median (range) & $54(15-73)$ \\
Right eyes & $17(68)$ \\
Phakic & $18(72)$ \\
No. of eyes with previous trabeculectomy & 5 \\
Preoperative visual acuity (logMAR) & $0.54(0.4-1.2)$ \\
Preoperative IOP & $30(26-38)$ \\
No. of eyes per type of glaucoma & \\
Primary open angle & 9 \\
Neovascular & 6 \\
Acute primary angle closure & 5 \\
Acute secondary angle closure & 1 \\
Pigmentary & 1 \\
Congenital & 2 \\
Steroid-induced & 1 \\
\hline
\end{tabular}

Values are presented as number, number (\%), or median (interquartile range) unless otherwise indicated.

$\log$ MAR $=$ logarithm of the minimum angle of resolution; IOP = intraocular pressure. preoperative IOP was 30 (26-38) mmHg. A mean (range) IOP decrease of $55 \%(33 \%-77 \%)$ was achieved resulting in a median postoperative IOP of $14 \mathrm{mmHg}(12-14)$, 18 months after the trabeculectomy $(p<0.0001$, two-tailed Wilcoxon matched-pairs signed rank test) (Table 2 and Fig. 2). Median (IQR) visual acuity improved from 0.54 ( 0.40 1.20 ) to $0.4(0.24-0.70) \log \mathrm{MAR}$ at 12 months $(p=0.0006$, two-tailed Wilcoxon matched-pairs signed rank test) (Table

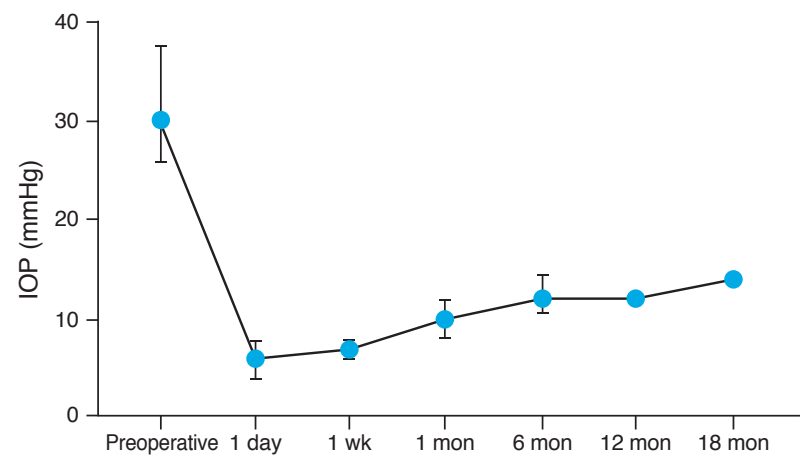

Fig. 2. Intraocular pressure (IOP). All values presented are median. Error bars are interquartile range.

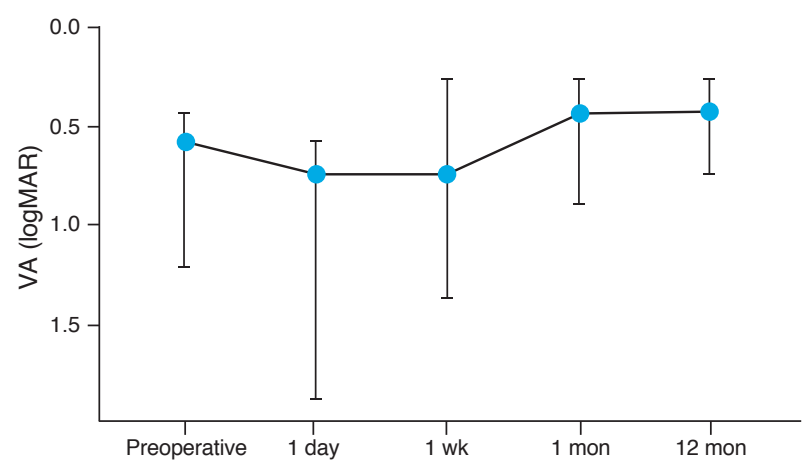

Fig. 3. Visual acuity (VA). All values presented are median. Error bars are interquartile range. $\log \mathrm{MAR}=$ logarithm of the minimum angle of resolution.

Table 2. Intraocular pressure and visual acuity assessments

\begin{tabular}{|c|c|c|c|c|c|c|c|}
\hline & \multirow{2}{*}{$\begin{array}{l}\text { Preoperative } \\
\text { assessment }\end{array}$} & \multicolumn{6}{|c|}{ Postoperative follow-up assessments } \\
\hline & & Day 1 & Week 1 & Month 1 & Month 6 & Month 12 & Month 18 \\
\hline \multicolumn{8}{|c|}{ Intraocular pressure } \\
\hline Median (IQR) & $30(26-38)$ & $6(4-8)$ & $7(6-8)$ & $10(8-12)$ & $12(10-15)$ & $12(12-14)$ & $14(12-14)$ \\
\hline$p$-value & & $<0.0001$ & $<0.0001$ & $<0.0001$ & $<0.0001$ & $<0.0001$ & $<0.0001$ \\
\hline \multicolumn{8}{|l|}{ Visual acuity } \\
\hline Median (IQR) & $0.54(0.40-1.2)$ & $0.70(0.54-1.80)$ & $0.7(0.24-1.30)$ & $0.4(0.24-0.85)$ & - & $0.4(0.24-0.70)$ & - \\
\hline$p$-value & & 0.007 & 0.681 & 0.006 & - & 0.001 & - \\
\hline
\end{tabular}

$\mathrm{IQR}=$ interquartile range. 
2 and Fig. 3). The median (IQR) number of glaucoma medication was reduced from 4 (3-4) to 0 after the operation. The operation was considered successful if IOP $\leq 15 \mathrm{mmHg}$ without need of IOP-lowering drops at 18 months. In our cohort, this was achieved in 21 out of the 25 eyes (84\%), the remaining four eyes all had IOP of 16 $\mathrm{mmHg}$ at 18 months. Short-term complication consisted of seven cases of bleb leakage treated with bandage contact lens and one case of mild choroidal effusion which settled with no intervention.

\section{Discussion}

Trabeculectomy has been the most common incisional surgery for treating glaucoma for more than 40 years [13]. Also, it is known that postoperative scarring is a major problem that affects the long-term success of trabeculectomy. Therefore, antimetabolite agents such as mitomycin- $\mathrm{C}$ were introduced to reduce fibroblast proliferation in the subconjunctival space and in Tenon's capsule and reduced the risk of scar formation [14]. But their side effects raised the prospect of several complications, such as corneal endothelial cell loss [15], cystic thin avascular bleb, choroidal detachment [16], endophthalmitis [17], and late-onset bleb leakage [18].

In our trabeculectomy technique, Ologen provides a space with a dynamic and physiological aqueous reservoir system because it is placed directly facing trabeculectomy opening and under the subconjunctival space. The implant influences the healing process by forcing the fibroblasts and myofibroblasts to grow into the pores and secrete a loose connective tissue matrix. Subsequently, Ologen is biodegraded by the body within 90 to 180 days from its implantation then ends by a fibrous meshwork structure which is almost similar to trabecular meshwork without its TM cells. Theoretically, this can potentially act as a space maintainer and a flow controller improving the surgical success of trabeculectomy [19].

Postoperative reduced IOP proved to be one of the important factors to preserve visual fields in these high-risk glaucoma patients. In the Advanced Glaucoma Intervention Study, it was found that in order to stop progress of glaucomatous visual field loss, postoperative IOPs in the low teens should be achieved [20]. Target pressures for normal pressure glaucoma patients might be even lower
[21]. It has been shown that reducing IOP by $30 \%$ in normal-pressure glaucoma patients diminishes the rate of visual field loss progression [22].

As earlier long-term studies often defined success as a postoperative IOP level of $21 \mathrm{mmHg}$ or less [23-25]. In our case series, a postoperative IOP level of less than $15 \mathrm{mmHg}$ was targeted in all patients. Our goal was to achieve a reasonable target IOP for trabeculectomies with the additional use of mitomycin-C and Ologen. Results of initial and follow-up assessment of IOP showed that the majority of our patients had IOP levels of $15 \mathrm{mmHg}$ or less at the end of the follow-up period. The percentage of IOP reduction on the short-term follow-up in our patients was 55\% (33\%$77 \%$ ). Further studies may be needed for long-term follow-up of IOP reductions in those patients.

It can be easily noted that our technique offers trabeculectomy with simple and straightforward steps which require basic surgical skills in the form of conjunctival and scleral flaps dissection with no scleral suturing which is always one of the tricky steps in trabeculectomy with the need for its optimum control to avoid being tight or loose and also any further need to be adjustable or releasable. This explains why the procedure is suitable for trainees with its very short learning curve. In our short-term study, none of our patients needed bleb needling, bleb revision, or suture lysis, which suggests that the procedure is a cost-effective procedure.

Furthermore, one of our goals was to achieve a cost effectiveness plan regarding the use of glaucoma medications. This was always a challenge in many studies as there was a gradual increase in the use of glaucoma medications in the follow-up period. In our case series, this could be attained during the follow-up of our patients.

Additionally, there was no deterioration of vision in our patients during the follow-up. Despite our study not comparing the visual field changes in these patients, we have maintained that IOP reductions and stability are the main factors in glaucoma progression.

The short-term results of our patients demonstrate that the procedure was of high safety profile with well-controlled short-term complications and no long-term complications during the follow-up period of the study. Reported early bleb leak in few patients was likely related to the closure technique which might be needed to be tighter in some patients but in those few patients. This early leakage was totally controlled by a bandage contact lens in the next 
few days following the procedure.

The current study faced a number of limitations that includes the relatively small sample size, follow-up time, and inability to compare our intervention with a matched control group. We consider this study to be a pilot study. Furthermore, we are planning to continue in a different study design with additional outcome measures.

Our case series study of those patients who underwent trabeculectomy with Ologen is aiming to find a safe and effective trabeculectomy procedure with a short learning curve. This study suggests that this technique can provide safety, effectiveness and a short learning curve for new ophthalmology trainees with lower incidence of operative and postoperative complications.

\section{Conflict of Interest}

No potential conflict of interest relevant to this article was reported.

\section{References}

1. Aung T, Tow SL, Yap EY, et al. Trabeculectomy for acute primary angle closure. Ophthalmology 2000;107:1298-302.

2. Bindlish R, Condon GP, Schlosser JD, et al. Efficacy and safety of mitomycin-C in primary trabeculectomy: fiveyear follow-up. Ophthalmology 2002;109:1336-41.

3. Fontana H, Nouri-Mahdavi K, Caprioli J. Trabeculectomy with mitomycin $\mathrm{C}$ in pseudophakic patients with open-angle glaucoma: outcomes and risk factors for failure. Am J Ophthalmol 2006;141:652-9.

4. Lamping KA, Belkin JK. 5-Fluorouracil and mitomycin C in pseudophakic patients. Ophthalmology 1995;102:70-5.

5. Mietz H, Raschka B, Krieglstein GK. Risk factors for failures of trabeculectomies performed without antimetabolites. Br J Ophthalmol 1999;83:814-21.

6. Molteno AC, Bosma NJ, Kittelson JM. Otago glaucoma surgery outcome study: long-term results of trabeculectomy. 1976 to 1995. Ophthalmology 1999;106:1742-50.

7. Sturmer J, Broadway DC, Hitchings RA. Young patient trabeculectomy. Assessment of risk factors for failure. Ophthalmology 1993;100:928-39.

8. Scott IU, Greenfield DS, Schiffman J, et al. Outcomes of primary trabeculectomy with the use of adjunctive mito- mycin. Arch Ophthalmol 1998;116:286-91.

9. Mermoud A, Salmon JF, Straker C, Murray AD. Post-traumatic angle recession glaucoma: a risk factor for bleb failure after trabeculectomy. Br J Ophthalmol 1993;77:631-4.

10. Negi AK, Kiel AW, Vernon SA. Does the site of filtration influence the medium to long term intraocular pressure control following microtrabeculectomy in low risk eyes? Br J Ophthalmol 2004;88:1008-11.

11. Megevand GS, Salmon JF, Scholtz RP, Murray AD. The effect of reducing the exposure time of mitomycin $\mathrm{C}$ in glaucoma filtering surgery. Ophthalmology 1995;102:84-90.

12. Five-year follow-up of the Fluorouracil Filtering Surgery Study. The Fluorouracil Filtering Surgery Study Group. Am J Ophthalmol 1996;121:349-66.

13. Lichter PR, Musch DC, Gillespie BW, et al. Interim clinical outcomes in the Collaborative Initial Glaucoma Treatment Study comparing initial treatment randomized to medications or surgery. Ophthalmology 2001;108:1943-53.

14. Lee DA, Shapourifar-Tehrani S, Kitada S. The effect of 5-fluorouracil and cytarabine on human fibroblasts from Tenon's capsule. Invest Ophthalmol Vis Sci 1990;31:1848-55.

15. Storr-Paulsen T, Norregaard JC, Ahmed S, Storr-Paulsen A. Corneal endothelial cell loss after mitomycin C-augmented trabeculectomy. J Glaucoma 2008;17:654-7.

16. Lim LA, Chindasub P, Kitnarong N. The surgical outcome of primary trabeculectomy with mitomycin $\mathrm{C}$ and A fornix-based conjunctival flap technique in Thailand. $J$ Med Assoc Thai 2008;91:1551-7.

17. Lama PJ, Fechtner RD. Antifibrotics and wound healing in glaucoma surgery. Surv Ophthalmol 2003;48:314-46.

18. Xu L, Wang Y, Li Y, et al. Causes of blindness and visual impairment in urban and rural areas in Beijing: the Beijing Eye Study. Ophthalmology 2006;113:1134.e1-11.

19. Yuan F, Li L, Chen X, et al. Biodegradable 3D-porous collagen matrix (Ologen) compared with mitomycin $\mathrm{C}$ for treatment of primary open-angle glaucoma: results at 5 years. J Ophthalmol 2015;2015:637537.

20. The Advanced Glaucoma Intervention Study (AGIS): 7. The relationship between control of intraocular pressure and visual field deterioration.The AGIS Investigators. $\mathrm{Am} \mathrm{J}$ Ophthalmol 2000;130:429-40.

21. Membrey WL, Bunce C, Poinoosawmy DP, et al. Glaucoma surgery with or without adjunctive antiproliferatives in normal tension glaucoma: 2 visual field progression. $\mathrm{Br} J$ Ophthalmol 2001;85:696-701.

22. Comparison of glaucomatous progression between untreat- 
ed patients with normal-tension glaucoma and patients with therapeutically reduced intraocular pressures. Collaborative Normal-Tension Glaucoma Study Group. Am J Ophthalmol 1998;126:487-97.

23. Diestelhorst M, Khalili MA, Krieglstein GK. Trabeculectomy: a retrospective follow-up of 700 eyes. Int Ophthalmol 1998-1999;22:211-20.
24. Mutsch YA, Grehn F. Success criteria and success rates in trabeculectomy with and without intraoperative antimetabolites using intensified postoperative care (IPC). Graefes Arch Clin Exp Ophthalmol 2000;238:884-91.

25. Parc CE, Johnson DH, Oliver JE, et al. The long-term outcome of glaucoma filtration surgery. Am J Ophthalmol 2001;132:27-35. 